\title{
Prevalence, correlates, and comorbidity related to attention-deficit hyperactivity disorder symptoms among Korean college students
}

\author{
Hangoeunbi Kang, M.D. ${ }^{1,2}$, Bo-Hyun Yoon*, M.D. ${ }^{1}$, PhD, Kyung-Min Kim, M.D. ${ }^{1}$ \\ Ha Ran Jung, M.D. ${ }^{1}$, Yu Ran Jeong, M.D. ${ }^{1}$, Hyun Ju Yun, M.D. ${ }^{1,2}$ \\ ${ }^{1}$ Department of Psychiatry, Naju National Hospital, Naju, Korea, ${ }^{2}$ Chonnam National University Medical School, Gwangju, Korea
}

Objectives: Attention-deficit hyperactivity disorder (ADHD) persists into adulthood in a high proportion of case. Adult ADHD is associated with several psychiatric comorbidities. Our study found that ADHD symptoms in adults were associated with several psychiatric comorbidities.

Aims: The aim of this study was to assess the prevalence of ADHD symptoms, the correlates, and the comorbidity among Korean college students.

Methods: A total of 2,593 college students participated in the study. Socio-demographic and clinical data were collected, as well as results from the Adult ADHD Self-Report Scale- Version 1.1 (ASRS-v1.1), the Center for Epidemiologic Studies Depression Scale (CES-D), the Korean version of the Mood Disorder (K-MDQ), a modified Korean version of the 16-item Prodromal Questionnaire (mKPQ), and the Alcohol Use Disorders Identification Test (AUDIT).

Results: ADHD symptoms were found in $4.7 \%$ of the participating college students. Univariate analysis revealed that female students had higher rates of ADHD symptoms than males $(p<0.001)$

We also found significant associations between ADHD symptoms and parental marital status, self-reported socioeconomic status, depression, bipolarity, psychosis risk, and problematic alcohol use ( $p<0.001$, respectively).

Multivariate analysis revealed that ADHD symptoms in college students were significantly associated with depression (OR $=5.94 ; 95 \% \mathrm{Cl} 3.65-9.69 ; \mathrm{p}<0.001)$, psychosis risk (OR =3.59; $95 \% \mathrm{Cl} 2.13-5.82$; $p<0.001$ ), bipolarity (OR $=2.15 ; 95 \% \mathrm{Cl} 1.02-$ 4.55; $p=0.045)$, and problematic alcohol use $(\mathrm{OR}=2.10,95 \% \mathrm{Cl}$ 1.35-3.27; $p=0.001$ ), after controlling for sex and age

Conclusion: Our study found that ADHD symptoms in adults were associated with several psychiatric comorbidities. These results suggested the need of early detection of ADHD symptoms in college students and emphasized the importance of implementing early psychiatric intervention to assess problems such as depression, psychosis risk, bipolarity, and problematic alcohol use in young adults with ADHD symptoms.

Table 1. Comparisons of characteristics among subjects with and without ADHD symptoms in Korean college students

\begin{tabular}{|c|c|c|c|}
\hline & $\begin{array}{l}\text { Non-ADHD symptoms group } \\
(\mathrm{N}=2463)\end{array}$ & $\begin{array}{l}\text { ADHD symptoms group } \\
(n=122)\end{array}$ & $p$ \\
\hline Age, years, $N(\%)$ & & & 0.634 \\
\hline$\leq 20$ & 1827(74.2) & $93(76.2)$ & \\
\hline $21-24$ & $532(21.6)$ & $26(21.3)$ & \\
\hline$\geq 25$ & $104(4.2)$ & $3(2.5)$ & \\
\hline Sex, N(\%) & & & $<0.001$ \\
\hline Male & 1133(46.0) & $35(28.7)$ & \\
\hline Female & $1330(54.0)$ & $87(71.3)$ & \\
\hline Parental martial status, N(\%) & & & $<0.001$ \\
\hline Married & $2135(87.7)$ & $89(76.1)$ & \\
\hline Unmarried(single, divorce, widowed) & $299(12.3)$ & $28(23.9)$ & \\
\hline Living arrangement, $\mathrm{N}(\%)$ & & & 0.791 \\
\hline Living with family & $1372(56.3)$ & $65(55.1)$ & \\
\hline Living alone & $1064(43.7)$ & $53(44.9)$ & \\
\hline Self-reported SES, N(\%) & & & 0.004 \\
\hline High & $221(9.0)$ & $12(10.2)$ & \\
\hline Middle & $1948(79.6)$ & $81(68.6)$ & \\
\hline Low & $279(11.4)$ & $25(21.2)$ & \\
\hline \multicolumn{4}{|c|}{ Mental health related condition (mean $\pm S D$ ) } \\
\hline CES-D (score) & $24.1 \pm 12.3$ & $9.8 \pm 8.7$ & $<0.001$ \\
\hline$M D Q$ (part 1 score) & $8.3 \pm 2.6$ & $5.2 \pm 3.2$ & $<0.001$ \\
\hline mKPQ (score) & $7.0 \pm 4.1$ & $2.7 \pm 2.8$ & $<0.001$ \\
\hline AUDIT (score) & $11.7 \pm 8.4$ & $8.4 \pm 6.1$ & $<0.001$ \\
\hline
\end{tabular}

Values are presented as meantstandad deviation or number(\%)

ADHD, Attention-Deficit Hyperactivity Disorder; SES, Social Economic Status; CES-D, The Center for Epidemiological StudiesDepression Scale; MDQ, The Mood Disorder Questionnaires; mKPQ, The modified Korean version of the 16-item Prodroma Questionnaire; AUDIT, The Alcohol Use Disorder Identification Test

Table 2. Comparisons of mental health related condition among subjects with and without ADHD symptoms in Korean college students

\begin{tabular}{|l|c|c|c|}
\hline & $\begin{array}{c}\text { ADHD symptoms group } \\
(n=122)\end{array}$ & $\begin{array}{c}\text { Non-ADHD symptoms group } \\
(\mathrm{N}=2463)\end{array}$ & \\
\hline Depression & & & $<0.001$ \\
\hline CES-D $>20$ & $73(61.9)$ & $280(11.5)$ & \\
\hline CES-D $\leq 20$ & $45(38.1)$ & $2159(88.5)$ & $<0.001$ \\
\hline Bipolarity (MDQ-respondents) & $15(12.9)$ & $39(1.7)$ & \\
\hline MDQ respondents & $101(87.1)$ & $2260(98.3)$ & \\
\hline MDQ non respondents & & & \\
\hline Early psychosis symptoms & $56(47.5)$ & $243(10.0)$ & \\
\hline mKPQ respondents & $62(52.5)$ & $2196(90.0)$ & $<0.001$ \\
\hline mKPQ non respondents & $54(46.6)$ & $578(24.1)$ & \\
\hline problematic alcohol use & $62(53.4)$ & $1816(75.9)$ & \\
\hline AUDIT $\geq 12$ & & & \\
\hline AUDIT $<12$ & & &
\end{tabular}

Table 3. Results of multivariate logistic regression analysis of association with ADHD symptoms in Korean college students

\begin{tabular}{|l|c|c|}
\hline Variables & OR $(95 \% \mathrm{Cl})$ & $\mathrm{p}$ \\
\hline Marital status of parents (ref: married) & & \\
\hline Separated (single, divorce, widowed) & $1.65(0.6-1.26)$ & 0.071 \\
\hline Self-reported SES (ref: high) & & \\
\hline Middle & $1.07(0.47-2.46)$ & 0.859 \\
\hline Low & $1.21(0.47-3.12)$ & 0.694 \\
\hline Depression (ref: no) & & \\
\hline Yes & & \\
\hline Early psychosis symptoms (ref: no) & $3.94(3.65-9.68)$ & $<0.001$ \\
\hline Yes & & \\
\hline Bipolarity (ref: no) & $2.15(1.02-4.55)$ & 0.001 \\
\hline Yes & & \\
\hline Problematic alcohol use (ref: no) & $2.10(1.35-3.27)$ & 0.001 \\
\hline Yes &
\end{tabular}

